\title{
New Low-Power Plasma Thruster for Nanosatellites
}

\author{
J. P. Sheehan*, Timothy A. Collard ${ }^{\dagger}$, Benjamin W. Longmier ${ }^{\ddagger}$, and Ingrid M. Goglio ${ }^{\S}$ \\ University of Michigan, Ann Arbor, MI, 48109, USA
}

\begin{abstract}
The CubeSat Ambipolar Thruster is a new, electrodeless, permanent magnet, helicon thruster specifically designed for the constraints of a nanosatellite. The design processes is outlined, indicating expected thrust, $\mathbf{I}_{\mathbf{s p}}$, and efficiency of $\sim 1 \mathrm{mN}, \sim 1000 \mathrm{~s}$, and $20 \%$, respectively. The major components of the thruster-quartz plasma liner, helical half-twist antenna, permanent magnets, and Faraday shield - are described. Finite element magnetic field simulations were compared to magnetometer measurements of the prototype device and showed agreement to within 5\%. Initial testing on xenon demonstrated ignition at $<50 \mathrm{~W}$. The electrodeless design enables the use of a wide variety of propellants and a discussion of iodine's potential as a propellant is included.
\end{abstract}

\section{Introduction}

GMALL satellites are changing the nature of spacecraft and space-based science. Their smaller size $(\leq 750$ $\mathrm{Nkg})$ allows them to be developed at a lower cost and on a more rapid development cycle. Nanosatellites $(1$ - $10 \mathrm{~kg}$ ) in particular have caught the attention of scientific and commercial entities alike for a wide range of tasks from imagery to space weather. ${ }^{1}$ The rapid emergence of nanosatellites has spawned the need for primary propulsion in these spacecraft. Chemical propulsion requires far more propellant mass than can be stored in such a small craft while conventional electric propulsion devices such as Hall thrusters and ion engines lose effectiveness when scaled down. This technological gap requires a new sort of electric propulsion device designed to be small $\left(\lesssim 1000 \mathrm{~cm}^{3}\right)$, low mass $(\lesssim 1 \mathrm{~kg})$, and low power $(\lesssim 50 \mathrm{~W})$. The CubeSat Ambipolar Thruster (CAT) described in this paper is designed specifically to fulfill these requirements.

\section{CubeSat Propulsion}

Since their introduction 10 years ago, CubeSats and their P-POD deployers ${ }^{2}$ have come to be the dominant nanosatellite design. A single CubeSat unit (U) is a cube with $10 \mathrm{~cm}$ sides with satellites consisting of some multiple or fraction of Us $(1 \mathrm{U}, 0.5 \mathrm{U}, 3 \mathrm{U}$, etc.). P-PODs are the deployment hardware that is attached to the launch vehicle, typically as secondary payload. It is specifically designed to protect the launch vehicle and primary payload while delivering the CubeSat(s) safely into orbit. Because of the adoption of this standard, construction costs have been reduced, standardized hardware can be manufactured, and flight heritage can be established across the many programs that use CubeSats. ${ }^{3,4}$ These programs include those of industry, universities, and countries without large space programs as well as large budget agencies such as NASA and the ESA.

CubeSat propulsion is less advanced than other nanosatellite technologies because of the difficulties in miniaturizing conventional thruster designs. Monopropellant and bipropellant systems exist or are in development $t^{5,6,7,8}$ with thrusts of $\lesssim 1 \mathrm{~N}$ and small $\Delta \mathrm{V}$ capabilities of $10 \mathrm{~s}$ of $\mathrm{m} / \mathrm{s}$. For moderate $\Delta \mathrm{Vs}(>100$ $\mathrm{m} / \mathrm{s}$ ) electric propulsion is needed. A wide variety of electric thrusters are in development, with predicted $\Delta \mathrm{V}$ capabilities up to $300 \mathrm{~m} / \mathrm{s} .{ }^{9}, 10,11,12,13,14,15,16,17$ These thrusters will be suitable for station keeping, formation flying, and deorbiting.

\footnotetext{
*Assistant Research Scientist, Aerospace Engineering, 1320 Beal Ave, Ann Arbor, MI 48109, AIAA Member.

${ }^{\dagger}$ Graduate Student, Aerospace Engineering, 1320 Beal Ave, Ann Arbor, MI 48109, AIAA Student Member.

¥Assistant Professor, Aerospace Engineering, 1320 Beal Ave, Ann Arbor, MI 48109, AIAA Member.

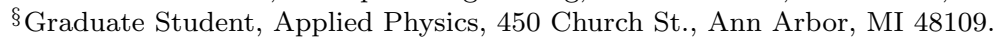

Copyright (C) 2014 by the American Institute of Aeronautics and Astronautics, Inc. The U.S. Government has a royalty-free license to exercise all rights under the copyright claimed herein for Governmental purposes. All other rights are reserved by the copyright owner.
} 
With a thruster capable of providing large $\Delta \mathrm{V}(>1 \mathrm{~km} / \mathrm{s})$ to a CubeSat a huge variety of new missions would become available. They would no longer be restricted to the orbit of the launch vehicle but could maneuver themselves into highly elliptical orbits, geostationary orbit, or polar orbits. With enough $\Delta \mathrm{V}$, CubeSats could reach the Earth-Moon or Earth-Sun Lagrange points. Significant cost savings would come from being able to deploy an array of satellites from a single launch vehicle, fanning them out to create a global network. Other possibilities include cluster formation flying and long-lived low altitude orbits.

\section{Helicon Thrusters}

Helicons are highly efficient plasma sources that are being explored for application in plasma propulsion. VASIMR is a $200 \mathrm{~kW}$ thruster that uses a helicon plasma source to heat the electrons and ion cyclotron resonance to heat the ions, both of which expand through a magnetic nozzle to produce thrust. ${ }^{18}$ This device has been designed for a next generation of spacecraft, being approximately $2 \mathrm{~m}$ long and requires far more power than conventional spacecraft can generate. Laboratory helicons are typically require power in the low $\mathrm{kW}$ range, which is on par with many established Hall thrusters and ion engines, and various groups have begun developing helicon thrusters on this scale. ${ }^{19,20,21,22}$ A miniature helicon thruster-HPH.com-is currently in development which uses only $10 \mathrm{~s}$ of $\mathrm{W}$ of power and has approximate performance parameters of $1 \mathrm{mN}$ of thrust, $1000 \mathrm{~s} \mathrm{I}_{\mathrm{sp}}$, and $20 \%$ efficiency. ${ }^{23,9}$

Helicon waves are bounded whistler waves, an electromagnetic plasma wave, which requires an externally applied magnetic field to propagate. ${ }^{24}$ They were discovered to be exceptionally good at coupling RF energy into a plasma to heat electrons and ionize neutrals, able to reach higher densities than capacitive or inductively coupled plasmas, making them useful as plasma sources. ${ }^{25,26,27,28}$ It is a low frequency wave ( $\omega \ll \omega_{c}$, where $\omega$ is the wave frequency, $\omega_{c}=\frac{e B}{m_{e}}$ electron cyclotron frequency, $e$ is the elementary charge, $B$ is the magnetic flux, and $m_{e}$ is the electron mass) so frequencies are typically industrial values of 6.78 , 13.56, or $27.12 \mathrm{MHz}$. The helicon dispersion relation at low frequency describes the wave's propagation in a plasma

$$
\frac{c^{2} k^{2}}{\omega^{2}}=\frac{\omega_{p}^{2}}{\omega \omega_{c} \cos \theta}
$$

where $c$ is the speed of light, $k$ is the wave number magnitude, $\omega_{p}=\sqrt{\frac{n_{e} e^{2}}{\epsilon_{0} m_{e}}}$ is the plasma frequency, $n_{e}$ is the electron number density, $\epsilon_{0}$ is the permittivity of free space, and $\theta$ is the angle between $\vec{k}$ and $\vec{B}$. The wave number can be decomposed into perpendicular and parallel components with respect the the magnetic field: $k^{2}=k_{\perp}^{2}+k_{\|}^{2}$. The perpendicular wave number depends only on the radius of the cylindrical discharge channel and the parallel wave number can be set by a proper antenna choice. ${ }^{29}$ Thus, for a system that fixes the RF frequency and the wave number, the density is proportional to the magnetic field strength, a result that has been validated often in experiments. ${ }^{27,30}$

\section{CubeSat Ambipolar Thruster}

\section{A. Design Drivers}

CAT was designed for use on a $3 \mathrm{U}$ CubeSat based on the Radio Auroral Explorer ${ }^{1}$ (RAX) or CubeSat investigating Atmospheric Density Response to Extreme driving (CADRE)bus, though it is suitable for almost any $3 \mathrm{U}$ CubeSat bus. The thruster itself was to be $1 \mathrm{U}$ or less, leaving $1 \mathrm{U}$ for propellant and $1 \mathrm{U}$ for spacecraft systems. This involved ensuring that the thruster was less than $1 \mathrm{~kg}$. Due to the design of the P-POD deployer there is some additional room inside where the deployment spring is curled. This so-called "tuna can" can allow the thruster to protrude slightly (up to $3.5 \mathrm{~cm}$ ) outside of the standard $3 \mathrm{U}$ shape. ${ }^{2}$

The RAX spacecraft design has four solar panels which generate a maximum of 7.0 or $8.1 \mathrm{~W}$ per panel, depending on the type of panel, and a $4.2 \mathrm{Ahr}$ battery pack. By charging the battery when the solar panels are illuminated by the sun the battery can sustain the spacecraft when it is shadowed by the earth. This power architecture can be used to CAT's advantage in certain missions. For instance, to raise the orbit apogee and increase the orbit eccentricity CAT can be fired near perigee during only a fraction of the orbit. In this way, CAT can operate at higher power than could be continuously provided by the solar panels. For this reason the nominal operating power of CAT was designed to be $10-50 \mathrm{~W}$, though larger operating power levels are possible, with demonstrations of up to $200 \mathrm{~W}$ with argon propellant. 


\section{B. Design of Key Parameters}

Table 1. CubeSat Ambipolar Thruster parameters for Xe propellant.

\begin{tabular}{|ll|}
\hline Power & $10-50 \mathrm{~W}$ \\
\hline Flow Rate & $5-15 \mathrm{sccm}$ \\
\hline Density (max) & $10^{14} \mathrm{~cm}^{-3}$ \\
\hline B Field (max) & $800 \mathrm{G}$ \\
\hline $\mathrm{I}_{\mathrm{sp}}$ & $400-800 \mathrm{~s}$ \\
\hline Efficiency & $10 \%-40 \%$ \\
\hline Thrust & $0.5-4 \mathrm{mN}$ \\
\hline$\Delta \mathrm{V}$ (see text) & $1-2 \mathrm{~km} / \mathrm{s}$ \\
\hline
\end{tabular}

The design starting point of CAT was the power. As an example, assume that $50 \mathrm{~W}$ gets deposited into the plasma by the antenna. Helicons are capable of producing nearly fully ionized plasmas and it takes some amount of energy per ion (called the ion cost, $W$ ) to sustain the discharge. This energy is an empirical number that accounts for ionization energy, wall losses, radiation, and all other energy sinks. The power balance equation can be written as

$$
\mathcal{P}=\frac{\dot{m}}{M} N_{a} W
$$

Where $\mathcal{P}$ is power, $\dot{m}$ is mass flow rate, $M$ is the molecular weight of the propellant, and $N_{a}$ is Avogadro's number. Using the ideal gas law, the mass flow rate can be re-expressed as a volumetric flow rate

$$
\dot{V}=\frac{\mathcal{P} R T}{P N_{a} W}
$$

where $\dot{V}$ is the volumetric flow rate, $R$ is the universal gas constant, and $P=1$ atm and $T=273 \mathrm{~K}$ for $\dot{V}$ units of sccm.

The ion cost depends on the propellant, but a typical number of $60 \mathrm{eV} /$ ion can be assumed for this example. In this case the volumetric flow rate is $12 \mathrm{sccm}$ which corresponds to $1.1 \mathrm{mg} / \mathrm{s}$ of xenon. The flow into the thruster must be balanced by flow out in steady state. The molecular rate into the thruster for full ionization is

$$
\dot{N}_{\text {in }}=\frac{\mathcal{P}}{W} .
$$

The molecular rate out of the thruster is determined by the plasma which exits at the sound speed $\left(c_{s}=\right.$ $\sqrt{T_{e} / m_{i}}$ where $T_{e}$ is the electron temperature and $m_{i}$ is the ion mass)

$$
\dot{N}_{\text {out }}=n_{e} c_{s} A_{e}
$$

where $A_{e}$ is the exit area. If an electron temperature of $10 \mathrm{eV}$ is assumed (which is reasonable in laboratory experiments) with xenon as the ion species then the electron density can be determined, which is $10^{14} \mathrm{~cm}^{-3}$ for CAT's exit area.

The necessary magnetic field strength for helicon wave propagation is given by solving the dispersion relation:

$$
B=\frac{e \mu_{0} n_{e} L_{A} \omega}{\pi \sqrt{\left(\frac{\pi}{L_{A}}\right)^{2}+\left(\frac{3.83}{a}\right)^{2}}}
$$

For CAT's antenna design the magnetic field strength is $\sim 800 \mathrm{G}$, the critical input for magnet design. As mentioned above, density is proportional to magnetic field strength, so higher magnetic field strength allows for higher thrust. Electromagnets require far too much power for practical use on a CubeSat, so permanent magnets were chosen.

The plasma inside the thruster is expected have electrons with a temperature of $5-20 \mathrm{eV}$ and cold ions, based on prior work. ${ }^{31,32}$ The electrons are magnetized and much faster than the ions, so they rush out of the thruster along the magnetic field lines. This establishes an electric field which accelerates the ions. This is known as ambipolar ion acceleration. ${ }^{32,33}$ The electrons cool in the acceleration region and transfer their thermal energy to ion directed kinetic energy. The initial electron temperature is much larger than the final temperature, so the ion energy can be approximated as

$$
E_{\mathrm{ion}}=\gamma T_{e}
$$

where $\gamma=2$ is the adiabatic index of this two degree of freedom system. ${ }^{33}$ From this equation, a rough estimation of $\mathrm{I}_{\mathrm{sp}}$ can be calculated: $400-800 \mathrm{~s}$ for xenon propellant. 
The electric power into the thruster $(\mathcal{P})$ goes into the ion kinetic energy or ion cost, which by definition includes all energy modes other than ion kinetic energy. Therefore, the maximum possible efficiency of the thruster $\left(\eta_{t}\right)$ is

$$
\eta_{t}=\frac{E_{\text {ion }}}{W+E_{\text {ion }}} \eta_{n}
$$

where $\eta_{n}$ is the nozzle efficiency and equals $\sim 0.9$ in CAT, using the conservative assumption of uniform density as a function of radius. As mentioned before, $W$ depends on both the propellant and the device, but values of $\sim 60 \mathrm{eV}$ are typical. ${ }^{18}$ Thus, the thruster efficiency is expected to be $10 \%-40 \%$. Thrust $(T)$ can then be calculated as

$$
T=\frac{2 \eta_{t} \mathcal{P}_{\mathrm{rf}}}{I_{\mathrm{sp}} g_{0}}
$$

where $g_{0}$ is Earth's gravitational acceleration. The thrust is expected to be $0.5-4 \mathrm{mN}$.

To estimate the $\Delta \mathrm{V}$ the CAT engine could provide, assume a $3 \mathrm{U}$ CubeSat where the thruster mass is 1 $\mathrm{kg}$, the propellant tank is $0.3 \mathrm{~kg}$, the propellant is $0.7 \mathrm{~kg}$, and the rest of the CubeSat is $1 \mathrm{~kg}$. With $\mathrm{I}_{\mathrm{sp}} \mathrm{S}$ between 400 and $800 \mathrm{~s}$, after using all of its propellant the satellite would have a $\Delta V$ of $1-2 \mathrm{~km} / \mathrm{s}$, large enough to enable many of the new missions mentioned above.

\section{Key Components}

Gaseous propellant flows from the propellant tank into the plasma liner, a quartz, bottle-shaped tube (see Fig. 1) where the gas is ionized into a plasma and heated. Quartz was chosen because of its low conductivity and ability to survive high temperatures. At pressures of ones of Torr the gas is on the border between free molecular flow and collisional flow, so just past the expansion point a "shower-head" gas distributor was included to disperse the gas into the liner in a more uniform radial profile. It has the added benefit of acting as an isolator, reducing the plasma that could propagate upstream and affect the valves and other components. The plasma liner's complex shape was manufactured by hand by a glass blower with a laser etcher used to create the shower-head holes. Such a process

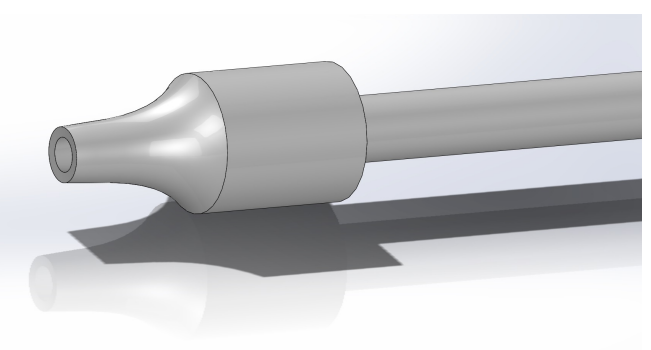

Figure 1. A model of the quartz liner, rendered in opaque material to better visualize the contours. does not scale well to manufacturing on even moderate scales, so in future designs other materials will be considered.

The liner diameter and nozzle were designed to optimize both the mass flow rate and the pressure in the liner. The flux of propellant into the liner should match the flux of plasma out to maximize propellant utilization. The pressure in the liner was chosen to provide the correct neutral density (i.e. equal to the plasma density). If the pressure was too high the electrons would collide before acquiring their maximum energy. If the pressure was too low they would traverse the entire plasma liner without making a collision. Even after the plasma is ignited, once the RF coupling becomes inductive or wave, the high energy ionizing electrons need to make their collisions within the liner for optimal performance.

The RF antenna was a helical half-twist geometry (see Fig. 2) designed to excite an $\mathrm{m}=+1$ helicon wave. The antennae for the initial experiments were $3 \mathrm{D}$ printed out of solid silver. Future designs will use electroplated silver on another stronger, less expensive metal, but solid silver allowed for rapid iteration of designs in the prototype phase. The wire diameter was $2 \mathrm{~mm}$ thick, balancing material cost with structural stability.

Although electromagnets are versatile, considering that the field strength can be

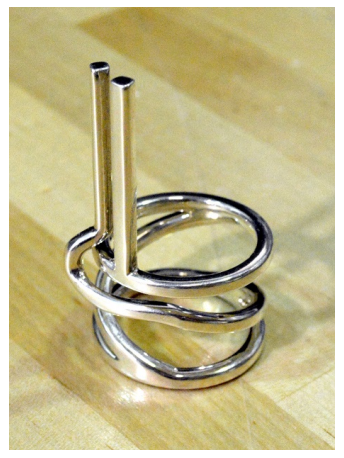

Figure 2. A helical half-twist antenna 3D printed out of solid silver. varied, they require a significant amount of power to operate. On CubeSats power is at an even greater premium than conventional satellites, so permanent magnets were used to generate the magnetic nozzle. This prototype used neodymium (NeFeB) rare earth magnets with strength 42 MGOe. Neodymium magnets suffer, though, from a relatively low Curie temperature of $\sim 150^{\circ} \mathrm{C}$, beyond which they become demagnetized. Samarium cobalt ( $\mathrm{SmCo}$ ) magnets are weaker, but have a higher Curie temperature 
$\left(\sim 300^{\circ} \mathrm{C}\right)$ and may be used in future versions of CAT. Carefully designed annular magnets were used to generate the magnetic nozzle which facilitates conversion of thermal energy to directed kinetic energy in the plasma. $^{32}$

The magnets, plasma liner, and antenna were contained and supported in a Faraday shield (see Fig. 3). The Faraday shield consists of two identical 3D printed titanium clam shells. Supporting the magnets is critical because the forces between them can be up to $50 \mathrm{~N}$. But the primary role of the shield is to contain the radio frequency energy from the antenna which could affect other parts of the spacecraft. Titanium was chosen because it is 3D printable, comparatively low density, high strength, and conductive so as to better contain the RF. The holes in the outer shell allow some observation of the interior for testing, but could be replaced with a solid version in an engineering model. Once assembled (see Fig. 3) the eight protruding legs get attached to rails appropriate for the specific CubeSat bus. The assembly shown was designed for integration with a RAX-like bus which will be the vehicle for the first on orbit test. The full assembly has a mass of $0.5 \mathrm{~kg}$, which does not include the RF power supply, the propellant, or tank.

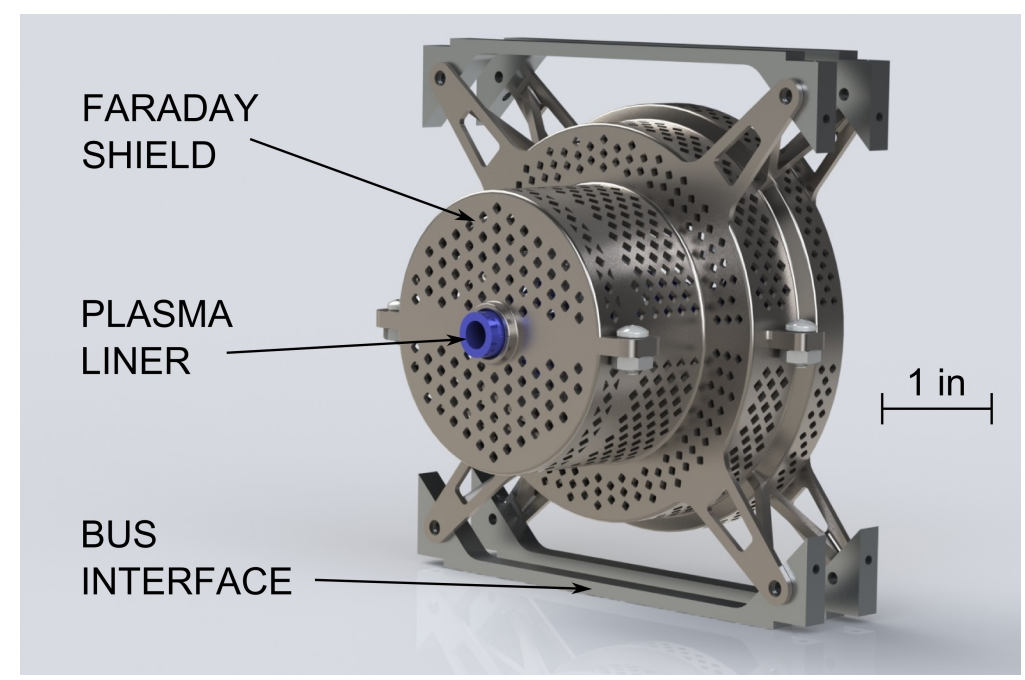

Figure 3. A CAD model of a fully assembled CAT engine. Enclosed is the liner, antenna, and magnets.

\section{Magnetic Field}

The magnets were designed using FEMM, a finite element magnetic field solver, to create an optimum magnetic field for CAT's operation. Once the thruster employing these magnets was constructed its magnetic field was measured using a three-axis magnetometer with $0.01 \mathrm{G}$ resolution. Because the magnetometer measurement head was larger than the plasma liner exit only the external magnetic field could be measured. Even if the liner was removed, simulations showed that the internal magnetic field varied significantly over distances of the size of the measurement head so internal measurements would be questionable at best.

The magnetic field was measured in a $40 \mathrm{~cm}$ by $50 \mathrm{~cm}$ grid with $0.5 \mathrm{~cm}$ spacing in front of the thruster (i.e. in the region of the plasma plume). The magnetic field lines from the measurements and from the simulations are overlain in Fig. 4. The measurements match the simulations very well- to within $5 \%$ (the percent deviation in field strength at a given point). Some of the variation comes from uncertainty in the direction in which the thruster was pointed and the absolute position at which the measurements were made. One particularly interesting feature of the measurements is at $40 \mathrm{~cm}$ axial distance from the thruster where the field line suddenly changes direction. This is the point at which CAT's magnetic field drops below Earth's magnetic field $(\sim 0.5 \mathrm{G})$ and Earth's magnetic field dominates. This measurement confirms the simulations' predictions that CAT's magnetic field has a practical extent of $40 \mathrm{~cm}$. This is critical for realistic thruster testing where the vacuum chamber wall effects can be minimized.

\section{E. Initial Testing}

A prototype CAT engine was assembled and tested in vacuum. The vacuum chamber was a $1 \mathrm{~m}$ diameter, $1 \mathrm{~m}$ long cylinder evacuated by a scroll pump and an CTI Cryogenics On-Board 10 cryopump which had a 


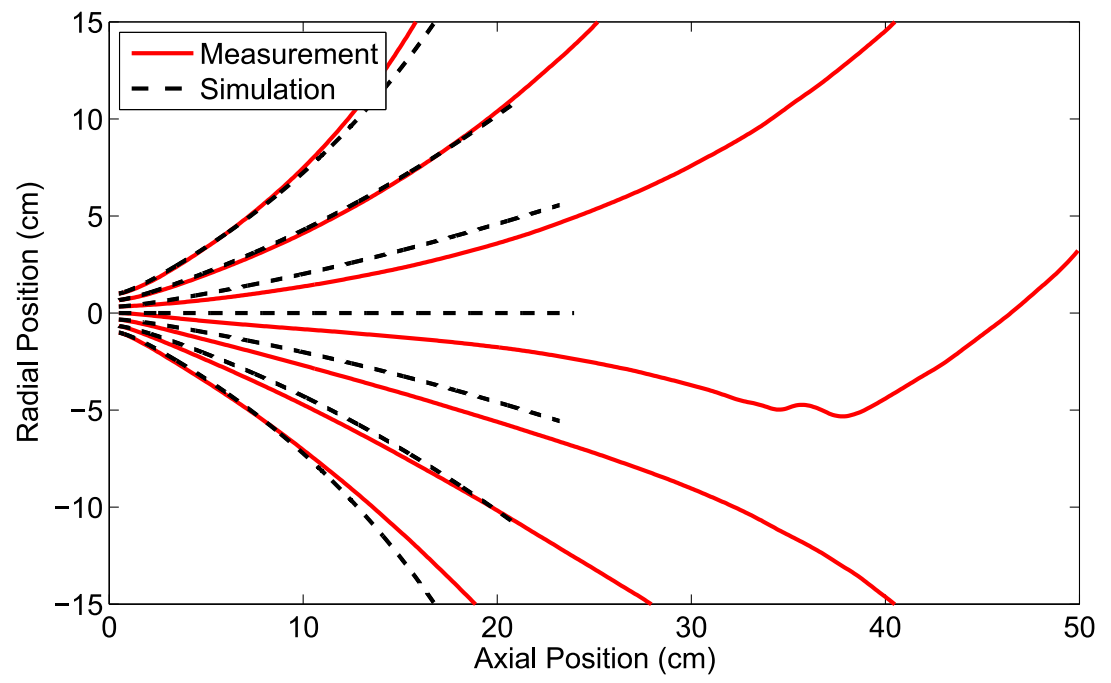

Figure 4. Magnetic field lines from simulations (dashed black) and measurements (solid red) downstream of the throat of the magnetic nozzle. The origin is the location of the throat of the nozzle.

pumping speed of $1500 \mathrm{l} / \mathrm{s}$ on air and $1200 \mathrm{l} / \mathrm{s}$ on argon. The chamber's base pressure was $\sim 10^{-6}$ Torr. The thruster was mounted to a stand on one end of the chamber. A two dimensional motion table using Velmex Xslide system hardware with $>5 \mu \mathrm{m}$ precision was used to move probes in the chamber.

Figure 5 shows a prototype CAT engine operating with xenon gas. The gas was fed from an external source into the plasma liner. The antenna surrounds the liner and was powered by an external $13.56 \mathrm{MHz}$ power supply with an L-type matching network. The external source offered more flexibility during the prototype and design phases. The Faraday shield was removed for this test in order to better see the plasma inside the liner. It is expected that the Faraday shield will greatly decrease the RF signal surrounding CAT but not seriously alter the magnetic field.

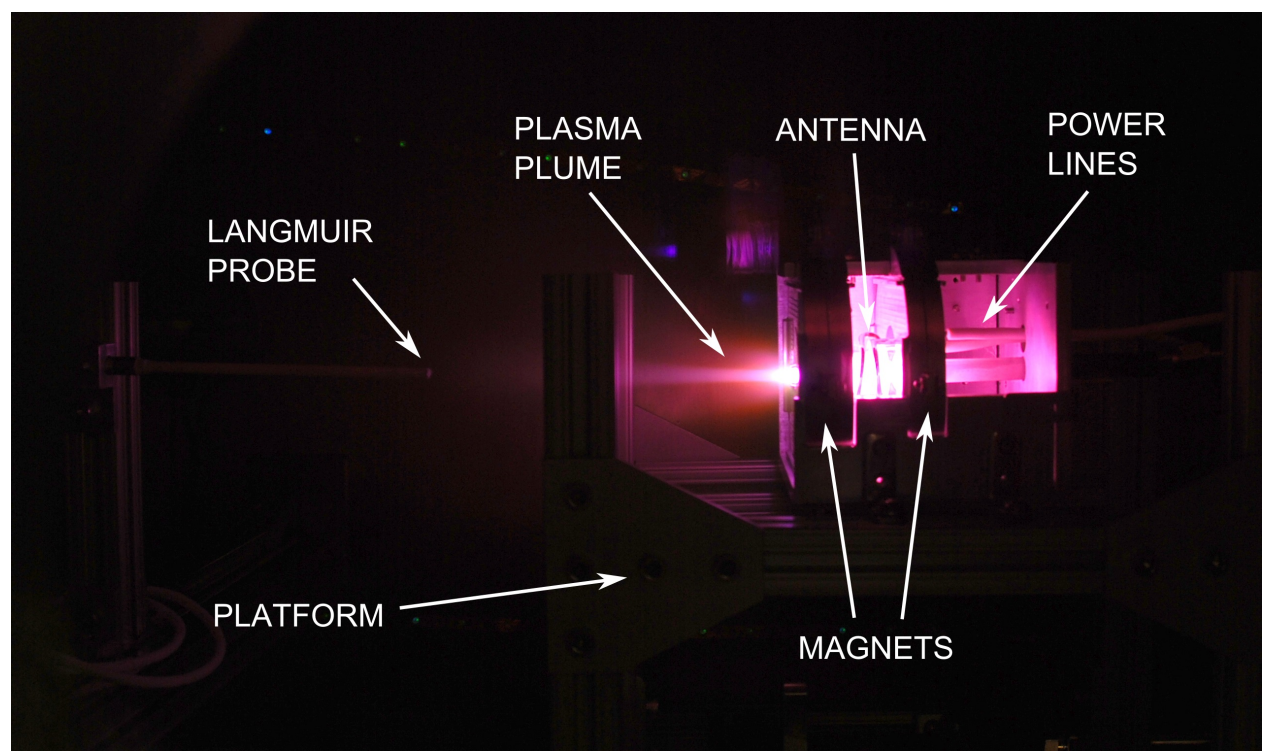

Figure 5. A photograph of a CAT prototype firing with xenon gas at $10 \mathrm{sccm}$ with $50 \mathrm{~W}$.

The thruster operation shown in Fig. 5 was with $10 \mathrm{sccm}$ of xenon and $50 \mathrm{~W}$ of power. The image of the 
thruster in operation confirms the antenna's ability to couple power into the plasma, though it is not clear at what efficiency. Some amount of power was lost to the transmission lines but that will not be an issue when the thruster design is complete, as the power supply will be adjacent to the antenna. The power that was coupled to the antenna generated the plasma which streamed out of the thruster along the magnetic field lines as indicated by the bright plume. The brightness is caused by electron collisions with neutrals and is correlated to the electron density, giving some initial indication that the electrons are following the magnetic field lines as expected.

\section{Propellants}

Central to CAT's design is that there are no electrodes exposed to plasma and the magnetic field keeps the plasma away from the plasma liner. These features allow CAT to be operated with not only conventional electric thruster propellants (xenon and krypton), but also novel propellants such as oxidizers or metals that would corrode or short circuit other thrusters. Xenon is most often used because it is inert and has a high mass, a low ionization potential, and a large cross section. Xenon, however, is becoming less economical and as a gaseous propellant has a low storage density. ${ }^{34,35,36,37}$ Solid- or liquid-storable propellants are of particular interest because of higher storage density, critical in CubeSats where volume is at a premium.

Iodine is a promising candidate due to its high storage density and

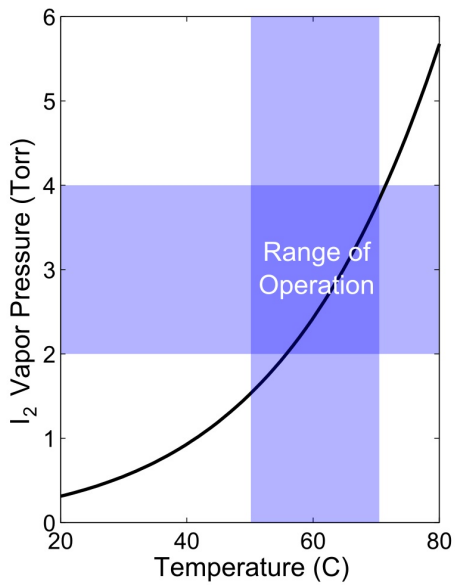

Figure 6. The vapor pressure of iodine as a function of temperature. low cost. Iodine and xenon have several advantageous similarities which may mean similar operation in CAT. ${ }^{34,35,36,37}$ They could provide similar thrusts and specific impulses because their atomic masses are so close (I: $126.9 \mathrm{amu}$; Xe: $131.3 \mathrm{amu})$. Iodine is a diatomic molecule as a solid and must be dissociated (1.54 eV dissociation energy) before the atoms can be ionized (10.5 eV ionization energy). The sum of these two energies is 12.0 $\mathrm{eV}$, very close to xenon's ionization energy of $12.1 \mathrm{eV}$. A key difference, though, is the rotation and vibration modes of $\mathrm{I}_{2}$ which could be an energy sink and reduce efficiency.

One of iodine's key features that makes it promising is its relatively high vapor pressures at temperatures reasonable for a spacecraft (see Fig. 6 ). To provide 1 Torr in the plasma liner, tank pressures need to be in the mid-1s of Torr which can be attained by heating the tank to $50-$ $70^{\circ} \mathrm{C}$. This can be achieved with a combination of passive heating and thermoelectric heaters. The feed lines will have to be heated to maintain the desired mass throughput.

Iodine is corrosive toward materials commonly found in satellites. This oxidation-reduction reaction tends to cause coating and breakdown of metals and brittleness or swelling in some elastomers. Table 2 outlines the compatibility of iodine with commonly used materials. The propellant feed modules being developed will utilize materials with high ratings, and component- and system-level tests will be conducted to verify the compatibility of the materials selected. The ratings were determined by visual observation over the course of up to 48 hours. For more information see Refs. 38 and 39. Iodine must be used with care, but in Hall thrusters have been successfully operated using it as a propellant, so it is a viable option for CAT. ${ }^{40}$

\section{Conclusion}

The CubeSat Ambipolar Thruster is an electrodeless plasma thruster designed for primary propulsion on CubeSats. A helicon wave couples RF energy into the plasma which accelerates through a magnetic nozzle. It has been designed to operate at up to $50 \mathrm{~W}$ and produce $\mathrm{mNs}$ of thrust with higher thrust levels possible (up to $200 \mathrm{~W}$ tested). Many of CAT's parts make use of 3D printing technology which allows for rapid iteration and the manufacture of precision parts that could not otherwise be machined. The magnetic nozzle was simulated using a finite element solver, the results of which were verified to within $5 \%$ of measured values. Using xenon, CAT was fired for the first time, providing a baseline for future testing. Some of these future tests will involve investigating iodine as a possible propellant. Iodine shows promise as a high performing, solid-storable propellant, enabled by CAT's electrodeless design. 
Table 2. Iodine compatibility of materials. ${ }^{38,39} \mathrm{~A}=$ Excellent - no effect. $\mathrm{B}=$ Good - minor effect, slight corrosion or discoloration. $\mathrm{C}=$ Fair - moderate effect, not recommended for continuous use. Softening, loss of strength, swelling may occur. $\mathrm{D}=$ Poor - not recommended for any use. Material ratings can vary depending on exposure times.

\begin{tabular}{cc}
\hline \hline Material & Rating \\
\hline 304 Stainless Steel & $\mathrm{D}$ \\
316 Stainless Steel & $\mathrm{D}$ \\
440 Stainless Steel & $\mathrm{D}$ \\
Aluminum & $\mathrm{D}$ \\
BUNA-N & $\mathrm{B}$ \\
Copper & $\mathrm{D}$ \\
EPDM & $\mathrm{B}$ \\
Epoxy & $\mathrm{C}$ \\
FKM & $\mathrm{A}$ \\
FFKM & $\mathrm{A}$ \\
Hastelloy-C & $\mathrm{A}$ \\
Kalrez & $\mathrm{A}$ \\
Nylon & $\mathrm{A}$ \\
PEEK & $\mathrm{C}$ \\
PTFE & $\mathrm{A}$ \\
Teflon & $\mathrm{A}$ \\
Titanium & $\mathrm{A}$ \\
Viton & $\mathrm{A}$ \\
\hline
\end{tabular}

\section{References}

${ }^{1}$ Cutler, J. W. and Bahcivan, H., "Radio Aurora Explorer: A Mission Overview," Journal of Spacecraft and Rockets, Vol. 51, No. 1, 2013, pp. 39-47.

${ }^{2}$ The CubeSat Program, C. P. S. I., "Cubesat Design Specifications Rev. 13," 2014.

${ }^{3}$ Robinson, R. M. and Moretto, T., "Small Satellites for Space Weather Research," Space Weather, Vol. 6, No. 5, 2008, pp. S05007.

${ }^{4}$ Swartwout, M., "University-Class Satellite: From Marginal Utility to 'Disruptive' Research Platforms," 2004.

${ }^{5}$ Schmuland, D., Masse, R., and Sota, C., "Hydrazine Propulsion Module for Cubesats," 2011.

${ }^{6}$ Schmuland, D., Carpenter, C., Masse, R., and Overly, J., "New Insights into Additive Manufacturing Processes: Enabling Low-Cost, High-Impulse Propulsion Systems," 2013.

${ }^{7}$ Dinardi, A. and Persson, M., "High Performance Green Propulsion (Hpgp): A Flight-Proven Capability and Cost GameChanger for Small and Secondary Satellites," 2012.

${ }^{8}$ Bertino-Reibstein, A. and Wuerl, A., "Development of a Warm-Gas Butane System for Microsatellite Propulsion," 2013.

${ }^{9}$ Trezzolani, F., Lucca Fabris, A., Pavarin, D., Selmo, A., Tsaglov, A. I., Loyan, A. V., Rubalov, O. P., and Manente, M., "Low Power Radio-Frequency Plasma Thruster Development and Testing," 2013.

${ }^{10}$ Abaimov, M. D., Sinha, W., Bilen, S. G., and Micci, M. M., "Cubesat Microwave Electrothermal Thruster (Cumet)," 2013.

${ }^{11}$ Shashurin, A., Keidar, M., and Zhuang, T., "Comparative Analysis of Micro-Cathode Arc Thruster Performance," 2013.

${ }^{12}$ Zhuang, T., Shashurin, A., Beilis, I., and Keidar, M., "Ion Velocities in a Micro-Cathode Arc Thruster," Physics of Plasmas, Vol. 19, No. 6, 2012, pp. 063501.

${ }^{13}$ Kronhaus, I., Schilling, K., Jayakumar, S., Kramer, A., Pietzka, M., and Schein, J., "Design of the Uwe-4 Picosatellite Orbit Control System Using Vacuum-Arc-Thruters," 2013.

${ }^{14}$ Fuchikami, S., Nakamoto, M., Toyoda, K., and Cho, M., "Development of Vacuum Arc Thruster for Nano Satellite," 2013.

${ }^{15}$ Di Cara, D., Bulit, A., Gonzalez del Amo, J., Romera, J. A., Leiter, H., Lauer, D., Altmann, C., Kukies, R., Polli, A., Ceruti, L., Antimiani, A., Feili, D., Lotz, B., and Serafini, L., "Experimental Validation of Rit Micro-Propulsion Subsystem Performance at Epl," 2013.

${ }^{16}$ Martel, F., Perna, L., and Lozano, P., "Miniature Ion Electrospray Thrusters and Performance Test on Cubesats," 2012.

${ }^{17}$ Spence, D., Ehrbar, E., Rosenblad, N., Demmons, N., Roy, T., Hoffman, S., Williams, D., Hruby, V., and Tocci, C., "Electrospray Propuslion Systems for Small Satellites," 2013. 
${ }^{18}$ Longmier, B. W., Squire, J. P., Olsen, C. S., Cassady, L. D., Ballenger, M. G., Carter, M. D., Ilin, A. V., Glover, T. W., McCaskill, G. E., Chang Daz, F. R., and Bering, E. A., "Improved Efficiency and Throttling Range of the Vx-200 Magnetoplasma Thruster," Journal of Propulsion and Power, Vol. 30, No. 1, 2014, pp. 123-132.

${ }^{19}$ Takahashi, K., Charles, C., Boswell, R. W., and Ando, A., "Helicon Plasma Thruster Experiment Controlling Cross-Field Diffusion within a Magnetic Nozzle," 2013.

${ }^{20}$ Shinohara, S., Nishida, H., Tanikawa, T., Hada, T., Funaki, I., and Shamrai, K. P., "Development of Electrodeless Plasma Thrusters with High-Density Helicon Plasma Sources," Ieee Transactions on Plasma Science, Vol. 42, No. 5, 2014, pp. 1245-1254.

${ }^{21}$ Tonooka, S., Funaki, I., Matsuoka, T., Iwabuchi, S., Nakamura, T., Shinohara, S., and Nishida, H., "Thrust Characteristics of Helicon Plasma Thrusters," 2013.

${ }^{22}$ Chen, F. F., "Performance of a Permanent-Magnet Helicon Source at 27 and 13 Mhz," Physics of Plasmas, Vol. 19, No. 9, 2012, pp. 093509.

${ }^{23}$ Pavarin, D., Ferri, F., Manente, M., Curreli, D., Guclu, Y., Melazzi, D., Rondini, D., Suman, S., Carlsson, J., Bramanti, C., Ahedo, E., Lancellotti, V., Katsonis, K., and Markelov, G., "Design of 50 W Helicon Thruster," 2009.

${ }^{24}$ Chen, F. F., "Helicon Plasma Sources," High Density Plasma Sources : Design, Physics, and Performance, edited by O. A. Popov, Noyes Publications, Park Ridge, N.J., 1995, p. 42.

${ }^{25}$ Boswell, R. W., "Very Efficient Plasma Generation by Whistler Waves near the Lower Hybrid Frequency," Plasma Physics and Controlled Fusion, Vol. 26, No. 10, 1984, pp. 1147-1162.

${ }^{26}$ Chen, F. F., "Plasma Ionization by Helicon Waves," Plasma Physics and Controlled Fusion, Vol. 33, No. 4, 1991, pp. 339-364.

${ }^{27}$ Chen, F. F., "Experiments on Helicon Plasma Sources," Journal of Vacuum Science and Technology a-Vacuum Surfaces and Films, Vol. 10, No. 4, 1992, pp. 1389-1401.

${ }^{28}$ Shoji, T., Sakawa, Y., Nakazawa, S., Kadota, K., and Sato, T., "Plasma Production by Helicon Waves," Plasma Sources Science and Technology, Vol. 2, No. 1, 1993, pp. 5-10.

${ }^{29}$ Porte, L., Yun, S. M., Arnush, D., and Chen, F. F., "Superiority of Half-Wavelength Helicon Antennae," Plasma Sources Science and Technology, Vol. 12, No. 2, 2003, pp. 287-293.

${ }^{30}$ Komori, A., Shoji, T., Miyamoto, K., Kawai, J., and Kawai, Y., "Helicon Waves and Efficient Plasma Production," Physics of Fluids B-Plasma Physics, Vol. 3, No. 4, 1991, pp. 893-898.

${ }^{31}$ Lafleur, T., Charles, C., and Boswell, R. W., "Characterization of a Helicon Plasma Source in Low Diverging Magnetic Fields," Journal of Physics D-Applied Physics, Vol. 44, No. 5, 2011, pp. 055202.

${ }^{32}$ Longmier, B. W., Bering, E. A., Carter, M. D., Cassady, L. D., Chancery, W. J., Diaz, F. R. C., Glover, T. W., Hershkowitz, N., Ilin, A. V., McCaskill, G. E., Olsen, C. S., and Squire, J. P., "Ambipolar Ion Acceleration in an Expanding Magnetic Nozzle," Plasma Sources Science and Technology, Vol. 20, No. 1, 2011, pp. 015007.

${ }^{33}$ Sheehan, J. P., Longmier, B. W., Bering, E. A., Olsen, C. S., Squire, J. P., Ballenger, M. G., Carter, M. D., Cassady, L. D., Daz, F. R. C., Glover, T. W., and Ilin, A. V., "Temperature Gradients Due to Adiabatic Plasma Expansion in a Magnetic Nozzle," Plasma Sources Science and Technology, Vol. 23, No. 4, 2014, pp. 045014.

${ }^{34}$ Rainer, D., Yu-Hui, C., and Dale, L., Propellant alternatives for ion and Hall effect thrusters, Aerospace Sciences Meetings, American Institute of Aeronautics and Astronautics, 2000, doi:10.2514/6.2000-602.

${ }^{35}$ Szabo, J., Pote, B., Paintal, S., Robin, M., Hillier, A., Branam, R. D., and Huffmann, R. E., "Performance Evaluation of an Iodine-Vapor Hall Thruster," Journal of Propulsion and Power, Vol. 28, No. 4, 2012, pp. 848-857.

${ }^{36}$ Kieckhafer, A. and King, L. B., "Energetics of Propellant Options for High-Power Hall Thrusters," Journal of Propulsion and Power, Vol. 23, No. 1, 2007, pp. 21-26.

${ }^{37}$ Tverdokhlebov, O. and Semenkin, A., Iodine propellant for electric propulsion - To be or not to be, Joint Propulsion Conferences, American Institute of Aeronautics and Astronautics, 2001, doi:10.2514/6.2001-3350.

${ }^{38}$ Cole-Parmer, "Chemical Compatibility Database," accessed Jul. 2014, http://www.coleparmer.com/ChemicalResistance.

${ }^{39}$ Supply, G. I., "Pump Compatibility Chart - Chemical Resistance Chart," accessed Jul. 2014, http://www.grainger.com/tps/pumps_chemical_compatibility.pdf.

${ }^{40}$ Szabo, J., Robin, M., Paintal, S., Pote, B., Hruby, V., and Freeman, C., "Iodine Propellant Space Propulsion," 2013. 